\title{
ABSTRACTS \\ from \\ TRANSACTIONS published in JAPANESE
}

(Pages refer to the Japanese originals of this volume unless otherwise noticed)

The Micro-Colorimetric Determination of Magnesium in Milk. (pp. 431 434): By Masayoshi Sa'to \& Kiichi Murata.

The principle of this method is based upon the observation of Kolthoff, that magnesium impart a pink color to alkaline solution of acridine sulphodye (Titan yellow) and this dye can be used for the colorimetric determination of small quantities of magnesium.

The outline of procedure is follows:

Five cc of milk are treated with dazol reagent, acetate mixture and uranium acetate. The coagulum is allowed to stand for about 5 minutes, then filtered off, and $5 \mathrm{cc}$ protein free filtrate is taken $25 \mathrm{cc}$ flask and calcium precipitated by mean of the Clark's method.

For the determination of magnesium $2 \mathrm{cc}$ of the supernant fluied are pipetted off into a $25 \mathrm{cc}$ flask, $5 \mathrm{cc}$ of $0.5 \%$ soluble starch added, then 0.4 cc of dye (Titan yellow) and $1.5 \mathrm{cc}$ of $0.4 \mathrm{~N}$. natrium hydroxide respectively.

The content are mixed throughly and compared Bürker colorimeter with magnesium standard.

Untersuchungen von Essigbakterien aus Formosa (VI). (pp. 435 455): Von Shosuke TANaka. (Government Research Institute, Formosa Japan.)

Untersuchungen über die Verwitterung der Eruptivgesteine I II.-Verwitterung der Basalte. (pp. 456 472): Von Mituru HARAdA. (Aus dem Institut für Geologie der Landwirtschaftlichen Hochschule Tottori, Japan.)

Die Ergebnisse der Untersuchungen über die Verwitterung der Basalte des San-in Gebietes in Japan sind folgende.

1. Der Profilen der Olivinbasalte sind 5 Stufen von nachstehender Beschaffenheit entnommen worden: (1) Frisches Gestein. (2) Verwitterte dünne Schicht über frischem Gestein. Der Basalt geht an der Oberfläche in eine $0,5 \sim 2 \mathrm{~cm}$ stark verwitterte gelblichgraue Schicht über: die Grenze zwischen 
den beiden ist scharf. (3) Graue Unterhorizont, der aus dem unter Texturerhaltung des Gesteins sehr stark chemisch umgewandelten, aber noch an Ort und Stelle befindlichen Verwitterungsprodukt besteht (über $500 \mathrm{~cm} \mathrm{Mö-}$ chtigkeit). Das Product entspricht dem Zersatz nach Harrassowitz. In diesem Horizont befinden sich weisse oder graue Spaltungsausfïllungsmassen. (4) Hellbräunlichgrauer Mittelhorizont, der nicht völlig die Gesteinstextur beibehalten hat und im etwas lockerem Zustande ist $(100 \sim 200 \mathrm{~cm}$ Dicke). Rötlichbrauner Oberhorizont. Humusarmer Boden (100 209 cm Dicke).

2. Beim Profil des Nephelinbasaltes gibt es folgende 4 Stufen : (1) Frisches Gestein. (2) Gelbliche dünne Verwitterungsschicht über dem Gestein. Die Grenze zwischen frischem Gestein und dieser Schicht ist scharf. (3) Hellkressfarbiger. Unterhorizont (über $200 \mathrm{~cm}$ Mächtigkeit). (4) Kressfarbiger Oberhorizont, der aus Lehm besteht $(100 \mathrm{~cm}$ Dicke).

3. Das Profil des Trachybasaltes hat folgende 4 Stufen: (1) Frisches Gestein. (2) Grauer Unterhorizont (einige $m$ Dicke). Dieser Horizont hat die Gesteinstextur beibehalten und verwittert nicht so stark wie der Unterhorizont des. Olivinbasaltes. Das frische. Gestein geht allmählich in das Verwitterungsprodukt über, und es besteht keine scharfe Grenz zwischen den beiden. (3) Hellbrauner Mittelhorizont. (80 cm Dicke). (4) Brauner Oberhorizont $60 \mathrm{~cm}$ Dicke).

4. Im Beginn der Verwitterung nimmt der si-Wert (nach Niggli) etwas $\mathrm{ab}$, und nachher wächst deutlich an. Der al-Wert wächst mit dem Fortschreiten der Verwitterung an. Die Spaltungsausfüllungsmasse hat den grössten ki- und al-Wert. Die Zunahme des fm-Wertes im Beginn der Verwitterung beruht auf einem Ansteigen des f-Wertes, während der $m$-Wert sich vermindert. Der f-Wert vermindert sich auch bei der weiteren Verwitterung. Die Verminderungen des c-, alk- und m-Wertes sind beim Olivinbasalt bedeutend, beim Trachybasalt und Unterhorizont des Nephelinbasaltes dagegen verhältnismässig geringer. Die Zunahme des $\mathrm{k}$ - und $\mathrm{m} / \mathrm{c}$ - Wertes zeigt, dass $\mathrm{Na}$ mehr als $\mathrm{K}$, und $\mathrm{Ca}$ mehr als $\mathrm{Mg}$ ausgewaschen wurde.

5. Aus dem Olivin- und Nephelinbasalt entstehen die sehr basenarmen ungesättigten Rotlehme. Dagegen entsteht aus dem Trachybasalt der basenreiche fruchtbare Braunlehm. Alle Horizonte enthalten kleine Mengen an freier Tonerde. (Die Überschüsse der Tonerde im 5\%igen KOH-Auszug sind $1 \sim$ $1,5 \%)$.

6. Die Zersätze und dünnen verwitterten Schichten über den Gesteinen enthalten bedeutende Mengen an salzsäurezersetzbarem Anteil, der nach dem ki-Wert dem Halloysit entspricht, und fast keinen Kaolinanteil. Die Oberhorizonte dagegen bestehen hauptsächlich aus Halloysit und Kaolinit. Es ist anzunehmen, dass beim Basalt zuerst keine Kaolinitisierung, sondern nur die Bildung von Halloysit erfolgt, und Kaolinit nachher durch die Veränderung 
des Halloysits entsteht. Die erste Umwandlung des Plagioklases, Augits und der Hornblende ist eine Halloysitisierung. Bei der Verwitterung des Bàsaltes ist die Kaolinitisierung sekundür.

7. Der Quotient ki im Oberhorizont vermindert sich beim Salzsđureauiszug $\left.(1,+\sim 1,)^{-}\right)$, dagegen nimmt er bei Bauschanalyse $z u(2,0 \sim 3,6)$. Die Ursache hierfür liegt darin, dass eine Zersetzung des Halloysits stattgefunden hat und die dabei entstandene Tonerde ziemlich beweglich ist, die Kieselsâure sich aber in Quarz umgewandelt hat.

8. Wurch die Elektrodialyse wurde 1 onerde mit Kieselsäure zusammen als Komplexionen, bei dem stark entbasten Verwitterungsprodukt von Anfang an, bei dem basenreichen Produkt aber erst nach der Entfernung der Basen, in die Kathodenkammer übergeführt. Bei der Elektrodialyse fand eine Zersetzung statt, in besonders hohem Masse bei dem basenreichen Verwitterungsprodukt. Daher kann das End $\mathrm{pH}$ und die Austauschneutralität nach Mattson nicht genau bestimmt werden. Für die durch Elektrodialyse wenig zersetzbaren Verwitterungsprodukte der Olivinbasalte nehmen diese Werte mit der Verwitterung zu.

Acids and Alcohols as a Nutrient of Monascus. (pp. 473 479): By Kikichi SATO and Iwao NArTo. (Laboratory of the Monopoly Búreou of Formosa, Japan.)

Studies on Amylosynthease. XXVII.-On the Amylosynthease of Heigher Plants which accumulate Starch. (pp. 480 483): By Toyosaku MinaqAWA. (Agr. Chemical Laboratory, Tokyo Imperial University.)'

Studies on Amylosynthease. XXVHI. - Studies on Reaction Velocity. (pp. 483 488): By Toyosaku MinagawA.' (Agr. Chemical Laboratory, Tokyo Imperial University.)

\footnotetext{
Über ein neues krìstallinisches Diterpen "Sciadopiten" atus dem Blatt- und $Z$ weigö1 von Sciadopitys verticillata $S$. et $Z$. 1. Mitt.-Über die Eigenschaften dés Sciadopitens. (pp. 489 494): Vón Kitsuji Nishida und Hidetake Uota.
} 
Sciadopitys verticillata S. et Z. (Schirmtanme) wächst nur in Südjapan, besonders im östlichen Zentral-Hodo am Kiso, auf Koyasan (daher japanische Name Koya-maki) und in seiner unmittelbaren Umgebung, selten auf Shikoku und Kyushu. Es wird als Baumholz und Möbelholz, besonders als Fassholz hoch geschätzt, und auch als Zierbaum in Tempelhöfen angepflanzt. Durch die schirmförmigen anstehenden, grossen Doppelnadeln wird es von allen übrigen Koniferen gut unterschieden.

Wir isolierten aus dem Blatt- und Zweigöl von Podocarpus macrophylla Don. zwei bis heute unbekannte Diterpene $\mathrm{C}_{20} \mathrm{H}_{32}$, die $\alpha$ - und $\beta$-Podocarpren ${ }^{(1)}$ benannt wurden. J. Kawamura(2) gibt an, $\alpha$-Podocarpren durch Mischprobe im ätherischen Oel aus Sciadopitys verticillata nachgeweisen zu haben. Bezüglich der weiteren Aufklärung unternahmen wir, $\alpha$-Podocarpren aus Schirmtanne infolge ihrer ausgedehenten Verbreitung und etwas grösserer Ausbeute zu gewinnen, dabei scheidet sich aber ein neuer Kristall ab, aus dem man ca $74 \mathrm{~g}$ in schuppenförmigen Kristallen von Smp. 95 96 $6^{\circ}$ nach vielmaligem Umkristallisieren erhält, so dass dieses Kristall mit Sicherheit von $\alpha$-Podocarpren oder zwei anderen kristallinischen Diterpen ${ }^{(2)}\left(\delta\right.$-Podocarpren von Smp. $65^{\circ}$ und Diterpen-X von Smp. $111 \sim 112^{\circ}$ ) unterschieden werden kann. Dieses neue Diterpen der Schirmtanne benannten wir zur Unterscheidung zweckmössig Sciadopiten.

Die Untersuchung des ätherischen Oels beweist den Unterschied der Bestandteile mit dem aus ein und derselben Pflanzenart abgetrennten. Diese Tatsache beruht auf verschiedenen Faktoren, z. B. Standort, Arten der Bäume, Klima, Hybridisation, Jahereszeit usw., und es äussern sich Zweifel, ob die so isolierten Präparate infolge einiger unbekannten Umlagerungen in der Pflanze selbst oder während der Extraktion chemisch verändert sind.

Baker und Smith $^{(3)}$ fanden früher im Destillationsrückstand des Oels von Phylloclandus rhomboidalis ein kristallinisches, farbloses Diterpen (Smp. 95 ${ }^{\circ}$, $\left.[\alpha]_{\mathrm{D}}+16,06^{\circ}\right)$, das mit Brom und Permanganat beständig ist und Phyllocladen benannt wurde. Dieses Diterpen ist bisher in seinen chemischen Eigenschaften nicht näher aufgeklärt worden.

E. J. Blakie ${ }^{(4)}$ erhielt bei Untersuchung des ätherischen Oels von Dacridium colensoi ein Diterpen von Smp. 91 ${ }^{\circ}$, das mit dem aus Oel von Dacridium biforme identisch ist und das früher Goudie ${ }^{(5)}$ und Aitken ${ }^{(6,7)}$ Dacren von Smp. $96^{\circ}$ bezêichneten, von dem weitere bemerkenswerte Resultate in seiner chemischen Beziehung noch nicht festgestellt wurden. In der Analyse und im übrigen Verhalten, z. B. $[\alpha]_{\mathrm{D}}$, Smp. von Hydrochlorid und $\mathrm{HCl}$-Spalt.Produkt, erinnert Sciadopiten ganz an Dacren, doch bleibt die Frage noch offen, ob es mit diesem identisch ist: das nach der Versetzung des Amylnitrits ausgeführte Produkt unterschied sich nämlich weder in der Kristallform noch in Zersetzungspunkt von dem Dacren. 
Isolierung des Sciadopiten.

Als Ausgangsmaterial wurde das ätherische Oel aus dem Blatt und Zweig von Sciadopitys verticillata S. et Z. benutzt: nach Entwässerung mit Natriumsulfat wurden auch andere Terpene gewonnene. 'Die Ausbuete betrug 0,248\%. Zur Isolierung des Sciadopitens wurde das Oel im Vakuum unter Einleitung von $\mathrm{CO}_{2}$-Gas, wie folgende Tabelle zeigt, fraktioniert:

\begin{tabular}{c|c|c|c|c||c|c|c|c|c}
\hline \hline Fraktion & $\begin{array}{c}\text { Druck } \\
(\mathrm{mm})\end{array}$ & $\begin{array}{c}\text { Tempera- } \\
\text { ture } \\
\left({ }^{\circ} \mathrm{C}\right)\end{array}$ & $\begin{array}{c}\text { Ausbeute } \\
(\mathrm{g})\end{array}$ & Farbe & Fraktion & $\begin{array}{c}\text { Druck } \\
(\mathrm{mm})\end{array}$ & $\begin{array}{c}\text { Tempera- } \\
\text { ture } \\
\left({ }^{\circ} \mathrm{C}\right)\end{array}$ & $\begin{array}{c}\text { Ausbeute } \\
(\mathrm{g})\end{array}$ & Farbe \\
\hline I & 18 & $56 \sim 57$ & 180 & farblos & VII & 2 & $125 \sim 139$ & 26.5 & hellgelb \\
II & 18 & $60 \sim 63$ & 289 & $\prime \prime$ & VIII & 1 & $139 \sim 143$ & 61 & $\prime \prime$ \\
I' & 19 & $60 \sim 62$ & 288 & $\prime \prime$ & IX & 1 & $143 \sim 150$ & 22.2 & $\prime \prime$ \\
II' & 20 & $62 \sim 72$ & 75 & $\prime \prime$ & X & 1 & $151 \sim 158$ & 94.5 & gelb \\
III & 16 & $73 \sim 75$ & 70 & hellgelb & XI & 1 & $159 \sim 161$ & 113.5 & $\prime \prime$ \\
IV & 3 & $106 \sim 112$ & 87 & $\prime \prime$ & XII & 1 & $161 \sim 163$ & 18.5 & $\prime \prime$ \\
V & 2 & $112 \sim 114$ & 85 & $\prime \prime$ & Rück- & & & 59 & gelbliche \\
VI & 2 & $115 \sim 122$ & 31.5 & $\prime \prime$ & stand & & & & Masse. \\
\hline
\end{tabular}

Unter Kühlung mit Eis der Fraktionen IX XII (über $145^{\circ}$ bei $1 \mathrm{~mm}$ : abdestillierte Anteile) wird der Kristall geliefert. Das so erstarrende Destillat wurde auf dem Nutsche möglich schnell abgesogen, und alsdann auf eine Tomplatte gestrichen, um anhaftende Oel zu beseitigen, dabei erhielt man das schuppenförmige Kristall ; die Ausbeute betrug $74 \mathrm{~g}$, entsprechend 4,98\% des ursprünglichen Oels. Es schmilzt nach zwei maligem Umkristallisieren aus Aether und Methanol bei $95 \sim 96^{\circ} \mathrm{C}$., wahrend von dem früheren Beobachter $^{(2)}$ ( $\boldsymbol{\delta}$-Podocarpren aus Sciadopitys verticillata) ein Schmelzpunktangabe von $50^{\circ}$ gemacht wurde. Zur Trennung des Diterpen-X von Smp. 111 112 wurde der Kristall wiederholt in Aether gelöst und durch Versetzung mit Alkohol fraktioniert, wobei aber Schmelzpunkt $\left(95 \sim 96^{\circ}\right)$ des so ausgeschiedenen Kristalles sich gleich erwies. Es führte uns zum Resultate, dass. das aus Blatt- und Zweigöl der Schirmtanne isolierte Diterpen eine einheitliche: neue Substanz ist, die wir Sciadopiten bezeichnen.

Eingenshaften des Sciadopitens.

Nach Umkristallisieren aus Aether und Alkohol oder nur aus Alkohol stellt es geruch- und farblose glänzende Tafeln oder Schuppen dar; sie sind leicht löslich in Aether, Chloroform, Benzol, Petroläther und Aceton. In Eisessig, Methanol und Alkohol löst es sich in der Hitze leicht, aber in der Kälte schwer, in Wasser, Natriumkarbonat- und Alkalilösung weder in der Kälte noch in der Hitze. Mit konz. Schwefelsäure wird es sofort gelb gefarbt und die Färbung verschwindet selbst nicht nach einigen Tagen. Es verschwindet sofort in ätherischer oder chloroformischer Bromlösung während es in 
Acetonlösung mit Kaliumpermanganat beständig ist. Die Reaktion von Nastjukow ist negativ.

Bestimmung der Jodzahl :

$0,2917 \mathrm{~g}$ Sciadopiten wurđen nach Hüblscher Methode mit alkoholischer Jod-Sublimatlösung über Nacht vor Licht geschützt stehen gelassen, dann der Ueberschuss des Jods mit $\mathbf{N} / 10$-Natriumthiosulfatlösung zurücktitriert ; heirbei verbrauchte man $21,78 \mathrm{ccm}$, während bei der Kontrolle $44,69 \mathrm{ccm}$ der Thiosuilfatlösung eingebracht wurden.

$$
\begin{gathered}
\text { J. } Z .=\frac{0,012693 \times 22,01 \times 100}{0,2917}=95,77 \\
\text { Ber. für } \quad C_{Z_{0}} \mathrm{H}_{32} \mathrm{~F} \quad 93.24 \\
{[\alpha]_{\mathrm{D}}^{20}=\frac{(+0,34) \times 6,3}{0,5 \times 0,3875}=+11,05 \text { (in Chloroform) }}
\end{gathered}
$$

$3,999 \mathrm{mg}$ Subst. gaben $12,930 \mathrm{mg} \mathrm{CO}$, und $4,365 \mathrm{mg} \mathrm{H}_{2} \mathrm{O}$.

$3,153 \mathrm{mg}$ Subst. gabsn $10,124 \mathrm{mg} \mathrm{CO}_{2}$ und $3,336 \mathrm{mg} \mathrm{H} \mathrm{H}_{2} \mathrm{O}$,

$$
\begin{array}{lllll}
\mathrm{C}_{20} \mathrm{H}_{32} & \text { Ber. } & \text { C } 88,15 \text { H } & 11,85 \% . \\
& \text { Gef. } & \text { C } 88,18 ; 88,07 & \text { H } & 12,21 ; 12,01 \% .
\end{array}
$$

Molekulargewichtsbestimmung.

$$
\begin{array}{cccc}
0,257 \mathrm{mg} \text { Subst. } & 1,925 \mathrm{mg} \text { Kampfer; } & \text { St } 18,0^{\circ} \\
\text { Mol. G=w. für } & \mathrm{C}_{20} \mathrm{H}_{32} & \text { Ber. } 272 \\
& \text { Gef. } 282
\end{array}
$$

Sciadopitennitrosochlorid.

$0,5 \mathrm{~g}$ Sciadopiten wurden mit $1 \mathrm{~g}$ Amylnitrit und $2 \mathrm{~g}$ Eisessig versetzt und in einer Költemischung abgekühlt, sodann $0,5 \mathrm{ccm}$ konz. Salzsäure tropfenweise hinzugesetzt, wodurch die Mischung grünliche Farbe annimmt. Nach einer Nacht wurde der ausgeschiedene Kristall abfiltriert und auf der Tonplatte gesammelt. Nach Umkristallisieren aus Chloroform und Aceton erhielt man farblose Kristallchen von Zersp. $127 \sim 128^{\circ}$.

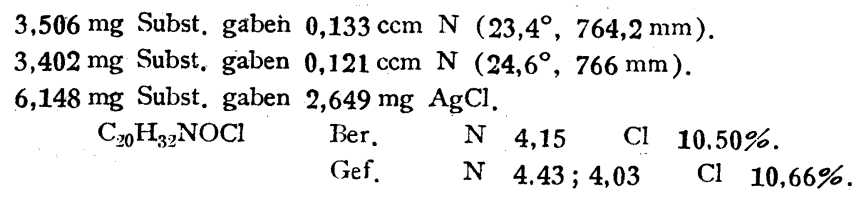

Sciadopitennitrosat.

$0,5 \mathrm{~g}$ Sciadopiten wurden mit $2 \mathrm{~g}$ Amylnitrit und $1 \mathrm{~g}$ Eisessig versetzt, dann wurde es mit Eiskochsalz gut erkaltet und dann kristallisierte nach einiger Zeit durch Zusatz einer Lösung von Salpetersäure, die $1 \mathrm{~g}$ konz. Salpetersäure auf $3 \mathrm{~g}$ Eisessig enthielt, das Sciadopitennitrosat in feinen Kristallchen aus, die nach dem Umkristallisieren aus Chloroform und Aceton als fein weisses kristallinisches Pulver den Zersp. 126 127 ${ }^{\circ}$ besitzen.

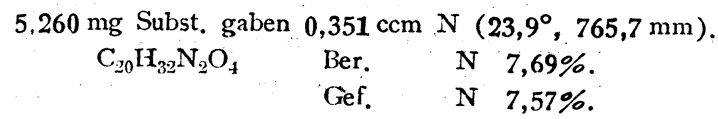


Sciadopitenmonohydrochlorid.

$2 \mathrm{~g}$ Sciadopiten wurden mit $30 \mathrm{ccm}$ trocknem Aether verdünnt und mit ganz getrocknetem Chlorwasserstoff unter starker Abkühlung gesättigt. Es bleibt über Nacht im Eiszimmer stehen, dann wurde Aether und überschüssiges Chlorwasserstoffgas unter vermindertem Druck abdestilliert. Der Rückstand wurde wieder in Aether gelöst, dann Methanol und Wasser zugefügt, wobei sich das Hydrochlorid in schönen farblosen glänzenden Schuppen abscheidet. Es fängt bei $101^{\circ}$ an zu sintern und schmilzt bei $106^{\circ}$ unter Schäumen und Zersetzung. In Aether, Chloroform, Petroläther, Benzol, Aceton und Essigester ist es sehr leicht löslich, in Eisessig, Methanol und Alkohol in der Kälte schwer löslich. Beim längerem Stehen vermindert es sich an Chlorwasserstoffgehalt und geht am Ende in ein Isomer des. Kohlenwasserstoffs über.

$$
\begin{aligned}
& {[a]_{\mathrm{v}}^{20}=\frac{(+0.17) \times 6.3}{0,2756 \times 0,5}=+7,77 \text { (in Benzöl) }} \\
& 4.470 \mathrm{mg} \text { Subst. gaben } 1,903 \mathrm{mg} \mathrm{AgCl} \text {. } \\
& 6,681 \mathrm{mg} \text { Subst. "gelen } 2,907 \mathrm{mg} \mathrm{AgCl} \text {. } \\
& \mathrm{C}_{20} \mathrm{H}_{32} \mathrm{HICl} \quad \text { Ber. } \quad \mathrm{Cl} 11,49 \% \text {. } \\
& \text { Gef. Cl 1n,53;10,79\%. }
\end{aligned}
$$

Salzsäureabspaltung aus Hydrochlorid.

$1,9 \mathrm{~g}$ Sciadopiten wurden mit $1,2 \mathrm{~g}$ Kaliumacetat in $30 \mathrm{ccm}$ Alkohol eine drittel Stunde lang am Rückflusskühler gekocht, der ausgeschiedene Kristall nach dem Erkalten filtriert und mit kaltem Alkohol gewaschen. Es kristallisiert aus dem heissem Alkohol in langen durchsichtigen Nadeln aus und erwies sich Smp. $106 \sim 107^{\circ}$, und w.urde Isosciadopiten benannt. Es ist in Aceton, Eisessig, Methanol und Alkohol in der Kälte leicht löslich, und in Wasser, Natriumkarbonat und Aetzalkalien weder in der Kälte noch in der Hitze. Mit konz. Schwefelsäure löst es sich .gelblich, gegen Permangant und Brom ist es beständig.

$$
[a]_{1)}^{20}=\frac{(+0,44) \times 6}{0,1193 \times 1}=+22,13^{\circ} \text { (in Chloroform). }
$$

$3,921 \mathrm{mg}$ Subst. gaben $12,661 \mathrm{mg} \mathrm{CO} 2$ und $4,181 \mathrm{mg}_{2} \mathrm{H}_{2} \mathrm{O}$.

$4,232 \mathrm{mg}$ Subst. gaben 13,658-mg $\mathrm{CO}_{2}$ und $4,547 \mathrm{mg} \mathrm{H}_{2} \mathrm{O}$.

$$
\begin{array}{llll}
\mathrm{C}_{20} \mathrm{H}_{32} & \text { Ber. } & \text { C } 88,12 & \mathrm{H} \\
& \text { Gef. } & \text { C } 88,12 ; 88,02 & \text { II } 11,93 ; 12,02 \% .
\end{array}
$$

Molekulargewichtsbestimmung.

$$
\begin{array}{ccc}
0,413 \mathrm{mg} \text { Subst., } & 5,161 \mathrm{mg} \text { Kampfer; } & \Delta t 11,0^{\circ} \\
\mathrm{C}_{20} \mathrm{H}_{32} & \text { Ber. } 272 & \text { Gef. } 276 .
\end{array}
$$

Einwirkung der Arneisensąure.

$1 \mathrm{~g}$ Sciadopiten wurde mit je $10 \mathrm{ccm}$ Ameisensaure und Alkohol versetzt und auf dem Wasserbade im Sieden 10 Stunden lang gekocht. Der nach 
der Abkühlen ausgeschiedene Kristall schmilzt bei $95 \sim 96^{\circ}$ und zeigt keine Schmelzpunktsdepression bei Mischprobe mit Sciadopiten. Sciadopiten wird also mit Ameisensäure nicht isomerisiert.

Isomerisierung des Sciadopitens mit schwetelsaure.

$1 \mathrm{~g}$ Sciadopiten wurde mit $20 \mathrm{ccm}$ Alkohol und $1 \mathrm{ccm}$ konz. Schwefelsäure versetzt und dann 5 Stunden am Rückflusskühler gekocht. Wir filtrierten das ausgeschiedene Kristall ab, waschen es mit kaltem Alkohol, kristallisierten dann wieder aus heissem Alkohol. Der so erhaltene Kristall bildet farblose lange Nadeln, dessen Schmelzpunkt bei $108 \sim 109^{\circ}$ liegt; er wird duch Mischen des durch Salzsäurespaltung aus dem Hydrochlorid dargestellten Präparats nicht erniedrigt. Es folgt aus diesem Befunde, dass es durch Schwefelsäure auch in Isosciadopiten isomerisiert werden kann, darüber wollen wir in der nächsten Mitteilung berichten.

Die folgende Tabelle zeigt die Schmelzpunkte und spez. Drehungsmögen des $\alpha$-Podocarprens, Sciadopitens und Dacrens.

\begin{tabular}{|c|c|c|c|}
\hline Schmelzpunkt & $\begin{array}{c}a \text {-Podocarpren } \\
30^{\circ}(1,:)\end{array}$ & $\begin{array}{l}\text { Sciadopiten } \\
95 \sim 96^{\circ}\end{array}$ & $\begin{array}{c}\text { Dacren } \\
91^{\circ(5)}, 96^{\circ(4)}\end{array}$ \\
\hline \multirow[t]{2}{*}{ Spez. Drehungsvermögen } & $\begin{array}{c}{[a]_{D}^{20}=-111,71^{\circ}} \\
\text { (in Chloroform(2)) }\end{array}$ & $\begin{array}{l}{[a]_{1}^{20}-+11.06^{\circ}} \\
\text { (in Chloroform) }\end{array}$ & $14,9^{\circ}(5), 10,7^{\circ}(4)$ \\
\hline & 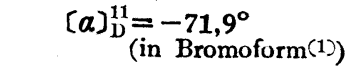 & & \\
\hline \multicolumn{4}{|l|}{ Hydrochlorid } \\
\hline Schmelzpunkt & $\begin{array}{l}114 \sim 115^{\circ}(1) \\
115 \sim 117^{\circ}(\mathbf{2})\end{array}$ & $106^{\circ}$ & $108,5^{\circ}(6)$ \\
\hline Spez. Drehungsvermơgen & {$[a]_{\mathrm{D}}^{20}=-27,4^{\circ}$ (in Benzol(1) } & {$[a]_{\mathrm{D}}^{20}=+7,77^{\circ}$} & $+10,9^{\circ}(6)$ \\
\hline \multicolumn{4}{|l|}{ HCl-Spaltungsprodukt } \\
\hline Schmelzpunkt & $\begin{array}{c}47 \sim 50^{\circ}(1) \& 197 \sim 199^{\circ}(1) \\
65^{\circ}(2)\end{array}$ & $\begin{array}{c}106 \sim 107^{\circ} \\
108 \sim 109^{\circ} \text { (mit } \mathrm{SO}_{4} \mathrm{H}_{2} \\
\text { isomerisiert) }\end{array}$ & $107^{\circ}(6)$ \\
\hline Spez. Drehungsvermögen & $\begin{array}{l}{[a]_{1}^{11}=-27,10} \\
\quad \text { (in Chloroform } ? 2)\end{array}$ & {$\left[a \stackrel{2}{1}_{\text {(in Chloroform) }}^{20}=+22.13^{\circ}\right.$} & $\left.+48,4^{\circ}: 6\right)$ \\
\hline \multicolumn{4}{|l|}{ Nitrosochlorid } \\
\hline $\begin{array}{l}\text { Zersetzungspunkt } \\
\text { Nitrosat }\end{array}$ & $136^{\circ(-)}$ & $127 \sim 128^{\circ}$ & Nicht kristallinisch \\
\hline Zersetzungspunkt & $134^{\circ} \& 140^{\circ}(2)$ & $126 \sim 127^{\circ}$ & Nicht kristallinisch \\
\hline
\end{tabular}

(1) K. Nishida und H, Uota: Bull. Agric. Soc. Japan. 6, 82 83 (1930); 7, 1 3 (1931);

7, 68 (1931):

(2) J. Kawamura: Bull. Imp. Forest. Exp. Sta., No. 31, 93 99 (1931).

(3) R. T. Barker and H. G. Sinith: A Research on the Pinus of Australia, Sydney, 3, 416 (1910).

(4) W, J. Blakie : J. Soc. Chem. Ind., 48, $357 \mathrm{~T}$ (1929).

(5) B. H. Goudie: J. Soc. Chem. Ind., 42, $357 \mathrm{~T}(1923)$.

(6) P. W. Aitken: J. Soc. Chem. Ind,, 47, 223 T (1928).

(7) W. J. Blakie: J, Soc. Chem. Ind., 49, 26 T (1930). 
On the Vitamin B Content of Milk powder Prepared by Merrelle-Soule process in Japan. (pp. 495 498): By Umetaro Suzuki, Sugao Hira) and Ryosuke IKEDA. (Agr. Chemical Laboratory, Tokyo Imperial University.)

On Systematic Study of Alcohol and Carbohydrate Oxidizing Bacteria isolated from Fruits, and a New Classification of the Oxidizing Bacteria (Continued). (pp. 499 513): By Toshinobu AsAI. (Agr. Ch.mical Laboratory, Tokyo Imperial University.)

On the Constituents of Rice-embryo It.-On Vitamin E in Riceembryo. (pp. 514 523): By Riang-Ha KIMM. (Institute of Physical and Chemical Research.)

On the Comparison of the Nutritive Value of Butter and Margarine (Preliminary Report). (pp. 524 532): By Ume Tange. (Institute of Physical and Chemical Research.)

Adding 15\% margarine in the diet as the source of fats, the rats develop the deficiency of vitamin A within about two months. Giving daily two or three drops of cod-liver oil, when not too advanced, the rats are cured in two weeks.

The inferiority of the nutritive value of margarine compared with that of butter is likely due to the deficiency of vitamin A rather than the nature of the fats, that is, at least the nutritive value of margarine is improved by adding vitamin $\mathrm{A}$.

Once given, vitamin A seems to be stored long in the body of rats, especially if vitamin $A$ is given when young and never administered afterwards, the rats do not show its deficiency at least for two months and half.

The nutritive value of vegetable hydrogenated fats is generally superior to that of fish hydrogenated fats.

On the Relationship between the Absorption of Fats and their Nutritive Value (Preliminary Report). (pp. 533 537): By Ume TANGE. (Institute of Physical and Chemical Research.) 
The main points in this study are :

A. To observe the growth of rats effected by various kinds of fats, with or without vitamin $B$ in the diet.

B. To see the relationship of the amount of fats, the rate of absorption and the growth of rats.

The results are summarized as follows :

1. In the diet without yeast, the growth of rats is best with the diet containing butter, then Criscs, lard and coco-nut oil, and the last, control (excluding fats), whereas with the diet containing $2 \%$ yeast, control has the condition of growth as good as the diet with butter.

2. With more fats in the diet, absorption increases, but the growth of rats is not parallel. For example, with cono-nut oil, ill effects are produced on the growth of rats. 